\title{
Problemas emocionais e comportamentais associados ao baixo rendimento acadêmico
}

\author{
Patricia Leila dos Santos \\ Sônia Santa Vitaliano Graminha \\ Universidade de São Paulo - Ribeirão Preto
}

\begin{abstract}
Resumo
Com o objetivo de identificar a incidência de problemas emocionais e comportamentais e os tipos de problemas de comportamento que aparecem associados ao desempenho escolar, aplicou-se a Escala Comportamental Infantil A2 e a Escala B de Rutter a dois grupos de crianças, um com baixo rendimento acadêmico (BRA) e outro com alto rendimento (ARA), cada grupo com 20 crianças, alunos de primeira e segundas séries, com idades que variaram entre 6 e 10 anos. Os resultados mostram que a maioria das crianças do grupo BRA obteve escores indicativos da presença de problemas emocionais/comportamentais, diferente do grupo ARA, e a incidência da maioria dos problemas específicos de comportamento foi sempre maior para o grupo BRA. Destaca-se que problemas de comportamento representam uma forte condição de risco para problemas de aprendizagem e que o trabalho com crianças com dificuldades de aprendizagem deve considerar aspectos ligados também ao comportamento.
\end{abstract}

Palavras-chave: problemas de aprendizagem; problemas de comportamento; fatores de risco; baixo rendimento acadêmico

\begin{abstract}
Emotional and behavioral problems associated to low academic performance. This study aimed to identify the incidence of emotional and behavioral problems, as well as the types of behavior problems associated to academic performance. For such purpose, Rutter's Child Behavior Scale-A2 and Rutter's B Scale were applied to students of first and second years of elementary school, aged 6 to 10 years and divided into two groups of 20 children each: a Low Academic Performance group (LAP) and a High Academic Performance group (HAP). The results evidenced that most of children in LAP group, unlike those in HAP group, reached scores indicative of the existence of emotional/behavioral problems, and that the incidence of the majority of the behavior-specific problems was always higher in LAP group. These findings highlight that behavioral problems represent a strong risk condition for learning problems and that working with children with learning disabilities should also take into account features related to behavior.
\end{abstract}

Keywords: learning disabilities; behavior problems; risk factors; low academic performance

A s associações entre distúrbios de comportamento e problemas de aprendizagem têm sido encontradas em vários estudos, sendo que alguns autores apontam as dificuldades de comportamento como sendo um fator de risco para o baixo desempenho acadêmico (Marturano, Linhares, \& Parreira, 1993; Speakman, Herman, \& Vogel, 1993; Thompson, Lampron, Johanson, \& Eckstein, 1990), enquanto outros indicam que problemas na aprendizagem escolar podem facilitar o desenvolvimento de distúrbios de comportamento graves (Gordon, 1993; Kolvin, Miller, Fleeting, \& Kolvin, 1988).

Roeser e Eccles (2000) propõem que as dificuldades comportamentais e emocionais influenciam problemas acadêmicos e estes afetam os sentimentos e os comportamentos da criança, sendo que estas dificuldades podem se expressar tanto de forma internalizada, por meio de ansiedade, depressão, retraimento e sentimentos de inferioridade, quanto externalizada, por meio de comportamentos e atitudes que geram conflitos com o ambiente e, geralmente, são marcados por características de desafio, impulsividade, agressão, hiperatividade e ajustamento social pobre.

A literatura aponta que as dificuldades de aprendizagem em si são uma condição de risco psicossocial, colocando o indivíduo em situação de desvantagem educacional e social. Pessoas que fracassam ou aquelas que abandonam a escola perdem oportunidades que poderiam representar experiências favorecedoras ao seu desenvolvimento. Assim, experiências escolares positivas e estimuladoras podem apresentar- 
se como condições que protegem o indivíduo de tornar-se delinqüente e envolver-se com a criminalidade (Kolvin et al., 1988; Maughan, 1988; Rutter, 1987).

Arnold et al. (2005), comparando adolescentes com e sem dificuldades de leitura quanto a problemas emocionais e comportamentais, colheram informações a partir de auto-relatos e dos relatos dos pais sobre sintomas apresentados. Os relatos dos adolescentes apontaram que aqueles com dificuldades de leitura apresentavam níveis mais altos de depressão, ansiedade e queixas somáticas do que os sem dificuldades. Os relatos dos pais mostraram mais problemas de atenção, queixas somáticas e comportamentos delinqüentes para os adolescentes com dificuldades de leitura.

De modo geral, os problemas de aprendizagem têm sido freqüentemente encontrados em associação com diferentes situações que envolvem tanto as características pessoais da criança com dificuldades quanto as condições de seu ambiente familiar e do ambiente mais amplo (Farran \& Cooper, 1986; Graminha, Martins, \& Miura, 1996; Lewis, Dlugokinski, Caputo, \& Griffin, 1988).

O conjunto de fatores de risco baseados na criança inclui componentes biológicos e de temperamento, manifestação de sintomas/problemas físicos e psicológicos e a competência (cognitiva e social) desenvolvida (Finnstrom, Gaddlin, Leijon, Samuelsson, \& Wadsby, 2003; Lewis et al., 1988). Comportamento é apenas um entre esses fatores; no entanto, merece destaque uma vez que os estudos sobre fatores de risco têm mostrado que os problemas de comportamento são condições importantes, freqüentemente associadas a distúrbios de aprendizagem ou baixo rendimento acadêmico.

Neste contexto, Speakman et al. (1993), por exemplo, apontam, entre os fatores de risco para problemas de aprendizagem, temperamento difícil, déficits de atenção, baixa tolerância à frustração e relacionamentos sociais pobres.

Em um estudo sobre a caracterização da clientela infantil com dificuldades escolares atendidas pelo serviço de psicopedagogia de um hospital-escola, Marturano, Magna e Murtha (1993) também encontraram resultados significativos apontando para a associação de dificuldades de aprendizagem com problemas de comportamentos; sejam aqueles diretamente ligados a relacionamentos e habilidades sociais ou aqueles relacionados à fala, ao sono, ao ritmo, às tarefas, aos hábitos, aos tiques e controle de esfíncteres.

Crianças com dificuldades de aprendizagem apresentam modos de enfrentamento inadequados frente às situações cotidianas e às relações interpessoais, predominando condutas que sugerem baixa capacidade de autoregulação, hostilidade e resistência às normas. Muitas vezes elas são descritas como desobedientes, irritáveis, impacientes, agitadas, inseguras, briguentas e destrutivas (Medeiros \& Loureiro, 2004).

Estudos têm demonstrado uma associação importante entre as dificuldades de aprendizagem e o auto-conceito da criança e a sua percepção de seus próprios problemas. Stevanato, Loureiro, Linhares e Marturano (2003) encontraram diferenças significativas quanto ao auto-conceito de crianças com dificuldades de aprendizagem comparadas a crianças com bom desempenho escolar, sendo que o grupo com dificuldades apresentou sempre resultados inferiores aos do grupo com bom desempenho. Estes dados são corroborados pelo estudo de Okano, Loureiro, Linhares e Marturano (2004), cujos resultados mostram que as crianças com dificuldades escolares se percebem com menor habilidade para aprender e com mais dificuldades de comportamento para ajustar-se às demandas do meio, quando comparadas às crianças sem dificuldades.

Witt, Riley e Coiro (2003), ao estudarem as associações entre status funcional da criança, estressores familiares e ajustamento psicossocial de escolares com diferentes dificuldades e limitações (perdas sensoriais, necessidades especiais, distúrbios de aprendizagem e outras), encontraram que problemas de comunicação e de aprendizagem, além de problemas de saúde física e mental da mãe, conflitos familiares e pobreza estão significativa e positivamente associados com desajustamento psicossocial entre crianças com dificuldades.

Passando para o contexto familiar, variáveis relacionadas às características pessoais dos pais, ao próprio funcionamento da família, à estrutura e à organização do ambiente doméstico aparecem associadas ao rendimento acadêmico. No contexto social mais amplo, encontram-se condições como a depreciação da criança por outras pessoas; rejeição e agressão; inadequação da professora; greve escolar e mudança de professora, classe ou escola durante o ano letivo. Estes fatores vão influenciar tanto a aprendizagem quanto o comportamento da criança em geral, podendo alguns deles aparecer desde a gestação e acompanhar por toda a sua vida (Graminha et al., 1996; Marturano et al., 1993; Santos, 2002; Vickers, 1994).

Santos e Graminha (2005), em um estudo sobre as relações entre o ambiente familiar e o rendimento acadêmico de crianças, encontraram um universo maior de adversidades familiares no contexto de crianças com baixo rendimento acadêmico. Segundo as autoras, esse contexto de adversidades vai se esboçando desde a gestação, continuando pelos anos pré-escolares e escolares. Apesar disso, elas apontam que as famílias de crianças com alto e baixo desempenho escolar podem ser muito semelhantes quanto à estrutura, organização e valores familiares, entretanto, têm condições de vida diferentes, sendo que as famílias de crianças que têm dificuldades na escola vivem em condições mais precárias e com menos conforto.

Outros eventos estressantes decorrentes de situações como hospitalizações ou institucionalização podem também representar uma fonte de risco para desordens emocionais/ comportamentais, bem como para problemas de aprendizagem (Farran \& Cooper, 1986; Lewis et al., 1988).

$\mathrm{Na}$ literatura sobre problemas de comportamento e aprendizagem, há indicações de que uma avaliação mais consistente sobre comportamento deve considerar tanto a percepção dos pais quanto a dos professores sobre a criança.

Silver (1981) realizou um estudo comparando três grupos de crianças, avaliadas por seus pais e professores por meio de escalas de comportamento: um grupo formado por crian- 
ças avaliadas pelo sistema escolar como tendo problemas de aprendizagem, outro por crianças diagnosticadas por pediatras como sendo hiperativas e/ou desatentas e um terceiro grupo que incluía crianças encaminhadas a um centro de saúde mental devido a problemas de comportamento. $\mathrm{O}$ autor encontrou que entre 26 e $41 \%$ das crianças com dificuldades na aprendizagem eram também hiperativas e/ou desatentas, 92\% daquelas classificadas como hiperativas e/ou desatentas tinham problemas de aprendizagem e, nestes dois grupos, $80 \%$ tinham problemas emocionais. Ele concluiu que essas crianças desenvolveram secundariamente problemas emocionais como uma conseqüência da frustração e deficiência em sua experiência. Postulou, então, que os problemas na aprendizagem e comportamentos caracterizados por hiperatividade e/ou desatenção favorecem o estabelecimento de problemas emocionais e/ou comportamentais.

Ainda com relação à avaliação comportamental, Haager e Vaughn (1995), em um estudo comparativo entre três grupos - o primeiro de crianças com distúrbios de aprendizagem, o segundo com baixo rendimento acadêmico e o terceiro com alto rendimento -, relataram que as crianças com distúrbio e as com baixo rendimento foram avaliadas por seus pais como tendo mais problemas de internalização e hiperatividade do que as com alto rendimento. Paralelamente, os professores avaliaram as crianças com distúrbios de aprendizagem e as com baixo desempenho como tendo mais problemas de internalização, hiperatividade e também mais problemas de externalização do que seus colegas com alto rendimento. Os autores concluíram que as diferenças na percepção de pais e professores tanto sobre comportamento quanto sobre habilidades sociais decorrem de uma diferença de perspectiva dos avaliadores. Essas diferenças precisam ser levadas em consideração nos processos de avaliação e na interpretação das informações sobre comportamento.

Como já foram expostos, os estudos sobre problemas de aprendizagem vêm repetidamente apontando uma associação entre dificuldades de comportamento e de aprendizagem, entretanto, não há consenso sobre a ordem de ocorrência destas dificuldades e se existe uma relação de causa e efeito entre elas. Além disso, a maioria dos estudos utiliza apenas uma fonte de informações, ou a família (pais) ou a escola (professores), apesar de a literatura apontar que, por terem perspectivas diferentes, é possível que a avaliação de pais e professores também seja diferente, tanto no que se refere a problemas de comportamento quanto de aprendizagem.

Assim, parecem particularmente importantes estudos que utilizem instrumentos semelhantes, aplicados tanto a pais quanto a professores, na investigação das associações entre comportamento e aprendizagem, buscando minimizar a influência das diferentes expectativas e possíveis vieses dos avaliadores.

Considerando estas questões, o presente trabalho tem por objetivo comparar as características comportamentais de dois grupos de crianças: um com baixo rendimento acadêmico e outro com alto rendimento, com base nas avaliações de pais e professores, buscando identificar em cada grupo a incidência de crianças com problemas emocionais e/ou comportamentais e os tipos de problemas de comportamento que aparecem associados ao desempenho escolar.

\section{Método}

Este estudo foi realizado numa escola pública estadual do interior do Estado de São Paulo, tendo como participantes 40 crianças de primeira e segunda séries do ensino fundamental, sendo que 20 delas foram classificadas por seus professores como tendo baixo rendimento acadêmico (grupo BRA) e 20 como tendo alto rendimento (grupo ARA).

Para a composição da amostra, foi solicitado aos professores que avaliassem todos os seus alunos (em um universo de 329 crianças de 12 classes diferentes), classificando-os numa escala de 1 a 10 (sendo 1 o mais baixo rendimento e 10 o mais alto rendimento). Foram então sorteadas 20 crianças classificadas nos pontos 1 e 2 para formar o grupo BRA e 20 classificadas nos pontos 9 e 10 para o grupo ARA.

Foram observadas as idades e séries e, para uma caracterização do nível de desenvolvimento das crianças, estas foram avaliadas por meio de três provas padronizadas: o Teste de Desempenho Escolar - TDE (Stein, 1994), o Teste de Matrizes Progressivas Coloridas (Raven, Raven, \& Court, 1988) e Teste Gestáltico Visomotor de Bender (Clawson, 1980).

No grupo BRA, as idades das crianças variaram entre seis anos e 11 meses e 10 anos e 10 meses, predominando meninos (75\%), alunos de segunda série (60\%), com desempenho escolar inferior ao esperado para a série freqüentada (95\%), nível intelectual médio, acima da média ou superior (55\%) e com desenvolvimento visomotor abaixo do esperado para a idade (60\%).

Por outro lado, entre as crianças do grupo ARA, as idades variaram entre seis anos e 11 meses e nove anos e 10 meses, predominando meninas (60\%), em igual proporção quanto à série freqüentada (50\% de primeira série e $50 \%$ de segunda série), com desempenho escolar marcadamente na média ou superior (100\%), nível intelectual também na média ou acima dela (90\%) e desenvolvimento visomotor dentro ou acima do esperado para a idade (100\%).

Para coletar informações sobre o comportamento das crianças foram utilizadas a Escala Comportamental Infantil A2 de Rutter (forma traduzida e adaptada por Graminha, 1994; 1998) e a Escala Infantil B de Rutter (Rutter, 1967), que foi traduzida especialmente para este estudo. A Escala A2 Adaptada é composta por 36 itens e foi desenvolvida para ser respondida pelos pais, enquanto a Escala B contém 26 itens, devendo ser preenchida pelo professor. Tomou-se o cuidado de realizar a coleta de dados no segundo semestre do ano letivo para que os professores pudessem conhecer melhor seus alunos, antes de avaliá-los. Das 40 mães a quem foi enviada a Escala A2, três não a devolveram devidamente preenchida; assim, os resultados referentes à Escala A2 consideram 37 participantes e os da Escala B referem-se a 40.

Os dados da Escala B foram computados de acordo com as orientações do autor (Rutter, 1967) e os dados da Escala 
A2 de acordo com o estudo de Graminha (1998). A partir das informações das duas escalas foram calculadas as porcentagens de alunos com escores totais indicativos de distúrbio emocional/comportamental para os grupos BRA e ARA, como também as porcentagens de ocorrência de cada item das escalas para os dois grupos.

Os resultados foram tabulados submetendo-se cada item da escala ao Teste Qui-quadrado de Pearson, ou ao Teste Exato de Fisher (quando o valor esperado era menor que cinco), utilizando-se o pacote estatístico SPSS. Foram considerados como significativos os resultados em que a probabilidade era inferior a 0,05. Também foi aplicado o teste de quiquadrado para verificar se a inclusão nos grupos BRA e ARA era independente de variáveis como sexo, idade, série freqüentada e a classificação obtida nos três testes aplicados.

Destaca-se que o estudo foi desenvolvido seguindose todos os cuidados éticos, esclarecendo a escola, as mães e as crianças sobre os objetivos e procedimentos a serem realizados, solicitando-se autorização dos pais para a avaliação das crianças e mantendo-se o sigilo sobre as informações coletadas.

\section{Resultados}

Inicialmente, na Tabela 1, é possível observar que os resultados da aplicação do qui-quadrado mostram-se estatisticamente significativos, indicando que o fato de a criança ser incluída em um dos dois grupos de estudo não independe do sexo e da classificação obtida nas três provas aplicadas.

Os resultados relativos às porcentagens de crianças dos grupos BRA e ARA que apresentam o problema descrito em cada item da Escala A2 Adaptada (respondida pelas mães) bem como os resultados do qui-quadrado ou do teste exato de Fisher, conforme o caso, são apresentados na Tabela 2.

É possível observar que, para a maioria dos itens da escala (31 dos 36 itens), a maior incidência ocorreu entre as crianças do grupo BRA. Os resultados foram significativos entre os dois grupos com relação aos itens: "fica mal humorado e nervoso”; “destrói suas coisas ou a dos outros”; "briga com outras crianças”; "não é querido pelas outras crianças”; ”criança fechada/solitária”; “não consegue permanecer numa atividade qualquer por mais do que alguns minutos”; “medo/receio de situações novas”; “criança difícil, complicada ou muito particular”; ”maltrata ou-

Tabela 1

Distribuição dos participantes por grupos (BRA e ARA), em referência ao sexo e às avaliações pelos testes de TDE, Raven e Bender (teste de qui-quadrado)

\begin{tabular}{|c|c|c|c|c|c|}
\hline & Variável & $\begin{array}{c}\text { Grupo BRA } \\
f\end{array}$ & $\begin{array}{c}\text { Grupo ARA } \\
f\end{array}$ & $\chi^{2}$ & $p$ \\
\hline \multirow[t]{2}{*}{ Sexo } & Masculino & 15 & 8 & & \\
\hline & Feminino & 5 & 12 & 5,013 & 0,025 \\
\hline \multirow[t]{2}{*}{ TDE } & Inferior & 19 & 0 & & \\
\hline & Médio / Superior & 1 & 20 & 36,190 & $<0,001$ \\
\hline \multirow[t]{2}{*}{ Raven } & Abaixo da média / Intelectualmente deficiente & 9 & 2 & & \\
\hline & Média / Acima da média / Superior & 11 & 18 & 6,144 & 0,013 \\
\hline \multirow[t]{2}{*}{ Bender } & Abaixo do esperado para a idade & 12 & 0 & & \\
\hline & Dentro ou acima do esperado & 8 & 20 & 17,143 & $<0,001$ \\
\hline
\end{tabular}

Tabela 2

Freqüências das respostas nos itens da Escala Comportamental A2 de Rutter Adaptada (para pais) entre os grupos BRA e ARA e testes estatísticos

\begin{tabular}{|c|c|c|c|c|}
\hline Item & $\begin{array}{c}\text { Grupo BRA } \\
\begin{array}{c}(\%) \\
\mathrm{N}=18\end{array}\end{array}$ & $\begin{array}{c}\text { Grupo ARA } \\
\text { (\%) } \\
\mathrm{N}=19\end{array}$ & $\chi^{2}$ & $p$ \\
\hline 1. Fica mal humorado e nervoso & 89 & 42 & 8,877 & 0,003 \\
\hline 2. Destrói suas coisas ou dos outros & 55 & 10 & 8,553 & 0,003 \\
\hline 3. Briga com outras crianças & 50 & 5 & $-{ }^{a}$ & 0,003 \\
\hline 4. Não é querido pelas outras crianças & 33 & 5 & $-{ }^{a}$ & 0,042 \\
\hline 5. Criança fechada/solitária & 55 & 16 & 6,414 & 0,011 \\
\hline $\begin{array}{l}\text { 6. Não consegue permanecer numa atividade } \\
\text { qualquer por mais do que alguns minutos }\end{array}$ & 61 & 21 & 6,153 & 0,013 \\
\hline 7. Medo/receio de situações novas & 50 & 10 & 6,894 & 0,009 \\
\hline 8. Criança difícil, complicada ou muito particular & 55 & 10 & 8,553 & 0,003 \\
\hline 9. Maltrata outras crianças & 28 & 0 & $-{ }^{a}$ & 0,020 \\
\hline 10. Fala palavrões & 67 & 10 & 12,386 & $<0,001$ \\
\hline 11. Insegura, não tem confiança em si mesma & 50 & 10 & 6,894 & 0,009 \\
\hline
\end{tabular}

a Para estes itens foi aplicado o Teste Exato de Fisher, obtendo-se diretamente o valor de $p$ 
tras crianças"; “fala palavrões” e "insegura, não tem confiança em si mesma”.

Complementando os dados obtidos por meio da Escala A2 (mães), a análise das respostas das mães à questão final da escala, que investiga se a criança apresenta outros problemas, mostrou que estas respostas foram afirmativas para 32\% das crianças do grupo BRA e $20 \%$ para as do grupo ARA.

Quanto aos resultados da Escala B (professores), na Tabela 3, é possível observar as porcentagens de crianças de cada grupo que apresentam o comportamento descrito nos diferentes itens da escala e os resultados dos testes estatísticos aplicados.

Os resultados da Escala B mostram que 23 dos 26 itens ocorreram com maior incidência para o grupo BRA, sendo que os resultados estatisticamente significativos ocorreram nos itens: "irrequieto / corre ou pula para cima e para baixo"; “mata aula”; "retorce-se/inquieto"; ”destrói suas próprias coisas ou a dos outros"; "briga com outras crianças”; ”não é querido pelas outras crianças”; "preocupado / preocupa-se com muitas coisas”; “irritável / perde o controle rapidamente”; "triste, infeliz, choroso ou angustiado"; "tende a estar ausente da escola por razões triviais”; “é desobediente”; “concentração pobre/curto período de atenção”; “conta mentiras”; “chorou / se recusou a entrar na escola este ano"; "gagueja”; "tem outra dificuldade de fala" e "maltrata outras crianças”.

Quando se comparam os resultados contidos nas Tabelas 2 e 3, chamam particularmente a atenção os dados relativos a seis itens semelhantes contidos nas Escalas A2 (para pais) e B (para professores) em que ocorreram resultados significativas entre os grupos BRA e ARA (com incidência maior no grupo BRA). A seguir, para facilitar a visualização, estes resultados aparecem resumidos na Tabela 4.

Complementando os resultados da Escala B (professores), a análise das respostas das professoras à questão final da escala que investiga se a criança apresenta outros problemas mostrou que estas respostas foram afirmativas para $20 \%$ das crianças do grupo BRA e $10 \%$ das do grupo ARA.

Tabela 3

Freqüencias de participantes por grupos (BRA e ARA) na Escala Comportamental B de Rutter (para professores) resultados nos testes estatísticos

\begin{tabular}{|c|c|c|c|c|}
\hline Item & $\begin{array}{c}\text { Grupo BRA } \\
(\%) \\
\mathrm{N}=20\end{array}$ & $\begin{array}{c}\text { Grupo ARA } \\
(\%) \\
N=20\end{array}$ & $\chi^{2}$ & $p$ \\
\hline 1. Irriquieto / corre ou pula para cima e para baixo & 45 & 5 & 8,533 & 0,003 \\
\hline 2. Mata aula & 25 & 0 & $-{ }^{a}$ & 0,047 \\
\hline 3. Retorce-se / inquieto & 50 & 5 & 10,157 & 0,001 \\
\hline 4. Destrói suas próprias coisas ou a dos outros & 30 & 0 & $-{ }^{a}$ & 0,020 \\
\hline 5. Briga com outras crianças & 65 & 5 & 15,824 & $<0,001$ \\
\hline 6. Não é querido pelas outras crianças & 35 & 5 & $-{ }^{a}$ & 0,044 \\
\hline 7. Preocupado / preocupa-se com muitas coisas & 5 & 50 & 10,157 & 0,001 \\
\hline 8. Irritável / perde o controle rapidamente & 55 & 0 & 15,172 & $<0,001$ \\
\hline 9. Triste, infeliz, choroso ou angustiado & 55 & 20 & 5,227 & 0,022 \\
\hline 10. Tende a estar ausente da escola por razões triviais & 50 & 0 & 13,333 & $<0,001$ \\
\hline 11. É desobediente & 35 & 0 & $-{ }^{a}$ & 0,008 \\
\hline 12. Concentração pobre / curto período de atenção & 95 & 5 & 32,400 & $<0,001$ \\
\hline 13. Conta mentiras & 35 & 5 & $-{ }^{a}$ & 0,044 \\
\hline 14. Chorou / se recusou a entrar na escola este ano & 30 & 0 & $-{ }^{a}$ & 0,020 \\
\hline 15. Gagueja & 25 & 0 & $-{ }^{a}$ & 0,047 \\
\hline 16. Tem outra dificuldade de fala & 35 & 0 & $-{ }^{a}$ & 0,008 \\
\hline 17. Maltrata outras crianças & 40 & 0 & $-{ }^{a}$ & 0,003 \\
\hline
\end{tabular}

${ }^{a}$ Para estes itens foi aplicado o Teste Exato de Fisher, obtendo-se diretamente o valor de $p$

Tabela 4

Distribuição porcentual de crianças em seis itens semelhantes das Escalas Comportamentais A2 (mães) e B (professores) em que ocorreram diferenças significativas entre os grupos BRA e ARA

\begin{tabular}{|c|c|c|c|c|c|}
\hline Item Escala A2 (mães) & BRA & ARA & Item Escala B (professores) & BRA & ARA \\
\hline Fica mal humorado e nervoso & 89 & 42 & Irritável / perde o controle rapidamente & 55 & 0 \\
\hline Destrói suas coisas ou dos outros & 55 & 10 & Destrói suas próprias coisas ou a dos outros & 30 & 0 \\
\hline Briga com outras crianças & 50 & 5 & Briga com outras crianças & 65 & 5 \\
\hline Não é querido pelas outras crianças & 33 & 5 & Não é querido pelas outras crianças & 35 & 5 \\
\hline $\begin{array}{l}\text { Não consegue permanecer numa atividade } \\
\text { qualquer por mais do que alguns minutos }\end{array}$ & 61 & 21 & $\begin{array}{l}\text { Concentração pobre / curto período de } \\
\text { atenção }\end{array}$ & 95 & 5 \\
\hline Maltrata outras crianças & 28 & 0 & Maltrata outras crianças & 40 & 0 \\
\hline
\end{tabular}


Finalmente, a Tabela 5 mostra os dados do grupo BRA e do grupo ARA, referentes aos escores totais obtidos por meio da Escala A2 de Rutter Adaptada (mães) e da Escala B (professores), indicativos da presença ou não de distúrbio emocional/comportamental.

Tabela 5

Distribuição percentual de crianças dos grupos BRA e ARA em função da ausência ou presença de distúrbios emocionais/comportamentais

\begin{tabular}{crr}
\hline \multirow{2}{*}{ Escalas } & \multicolumn{2}{c}{$\%$} \\
\cline { 2 - 3 } & BRA & ARA \\
\hline Escala A2 & & \\
Ausência de distúrbio emocional / comportamental & 39 & 79 \\
Presença de distúrbio emocional / comportamental & 61 & 21 \\
Escala B & & \\
Ausência de distúrbio emocional / comportamental & 30 & 5 \\
Presença de distúrbio emocional / comportamental & 70 & 95 \\
\hline
\end{tabular}

Comparando-se os resultados, é possível observar que a porcentagem de crianças do grupo BRA que apresenta escores indicativos de distúrbios emocionais e/ou comportamentais na Escala A2 (escore total maior que 16, conforme padronização brasileira realizada por Graminha, 1998) é quase três vezes maior do que a do grupo ARA (61\% contra 21\%). Esta diferença é ainda maior quando se observam os resultados da Escala B: a porcentagem de crianças do grupo BRA com escores indicativos de distúrbios emocionais e/ou comportamentais é 14 vezes maior do que a do ARA (70\% contra $5 \%$ ).

\section{Discussão}

Este trabalho se propôs a comparar dois grupos de crianças - um com alto e outro com baixo rendimento acadêmico - buscando observar associações entre comportamento e rendimento acadêmico, considerando dois informantes: pais e professores.

Retomando este objetivo, parece particularmente importante comentar os dados referentes à caracterização dos sujeitos, que apontam diferenças entre os dois grupos de crianças que vão além do rendimento acadêmico avaliado pelo professor.

A análise estatística ratificou que o baixo rendimento acadêmico foi mais freqüente entre os meninos; em contrapartida, as crianças com alto rendimento foram na maioria meninas. Além disso, os dados referentes às características das crianças também sugerem que aquelas que apresentam baixo rendimento acadêmico estão em desvantagem de desenvolvimento, uma vez que no grupo BRA foram significativamente maiores do que no grupo ARA: a incidência de crianças com desempenho escolar inferior ao esperado para a série freqüentada, o número de crianças com inteli- gência abaixo da média ou intelectualmente deficientes e o número de crianças com resultados visomotores abaixo do esperado para sua idade.

A diferença quanto ao sexo encontrada entre os dois grupos, apontando que baixo rendimento acadêmico é mais comum entre meninos que entre meninas, reafirma resultados apontados na literatura sobre maior incidência de problemas de aprendizagem entre meninos (Meister et al., 2001; Needleman, Salamanco, Murno, Lamy, \& De Gentile, 1996; Rutter et al., 2004).

Rutter et al. (2004), rebatendo críticas de que a alta taxa de problemas na leitura entre garotos era decorrente de distorções na interpretação sobre as dificuldades apresentadas pelos meninos, relatam os resultados de quatro grandes estudos realizados na Nova Zelândia e na Inglaterra, mostrando que em todos eles a diferença entre sexos se repetiu, confirmando que os meninos são mais freqüentemente atingidos por dificuldades de leitura.

Complementarmente, Barbaresi, Katusic, Colligan, Weaver e Jacobsen (2005), investigando a incidência de problemas de aprendizagem da matemática entre escolares, também encontraram que meninos são mais freqüentemente atingidos pelo problema do que as meninas.

No que se refere às características de comportamento das crianças, os resultados apontam, como outros estudos da literatura (Graminha, 1992; Hinshaw, 1992; Marturano et al., 1993), para uma forte associação entre problemas de aprendizagem e problemas emocionais e comportamentais.

Os resultados indicam uma coerência entre as avaliações de mães e professoras sobre o comportamento das crianças, uma vez que na grande maioria dos itens comuns às duas escalas utilizadas e, em especial, nos itens em que houve diferença significativa entre os dois grupos, os resultados mostram a mesma tendência (com maior incidência para o grupo BRA), diferente das observações feitas por Haager e Vaughn (1995) que indicaram diferenças nessas avaliações. É possível que a utilização de dois instrumentos desenvolvidos a partir de um mesmo referencial teórico, constituídos por itens semelhantes, tenha sido favorável às avaliações, moderando a interferência das diferentes expectativas dos avaliadores.

Os dados referentes ao comportamento avaliado pelas mães sugerem uma tendência maior das crianças com BRA a apresentarem tanto problemas relacionados à internalização quanto à externalização de comportamentos. É importante lembrar que alguns dos comportamentos apresentados com maior incidência pelo grupo BRA relacionam-se a problemas como hiperatividade, impulsividade e falta de atenção/concentração, características estas prejudiciais à aprendizagem escolar. Estes dados se assemelham aos de Magna (1997) referentes a comportamentos de crianças com problemas de aprendizagem durante o período pré-escolar.

Quando comparados com os dados do estudo de Graminha (1992), é possível observar que, com relação à maioria dos itens da Escala A2 de Rutter Adaptada, as incidências encontradas neste estudo referentes ao grupo BRA são 
maiores do que as encontradas pela autora no grupo de escolares que estavam atrasados na escola. Por outro lado, os dados relativos às crianças do grupo ARA são frequentemente próximos ou menores do que os encontrados por Graminha (1992), tanto quando os comparamos com os resultados do grupo que freqüentava a série escolar correspondente à idade cronológica, quanto com os do grupo de crianças adiantadas na escola. Esta diferença nos resultados dos dois estudos pode ser devida, primeiro, à grande diferença no tamanho das amostras, uma vez que os dados do trabalho referido resultam de um estudo epidemiológico; segundo, que, embora um dos grupos de crianças estudadas pela autora estivesse atrasado na escola, não significa que essas crianças estavam apresentando problemas quanto ao aprendizado ou rendimento escolar. Além disso, as crianças do grupo ARA, embora estejam se saindo bem na escola, estão freqüentando a série escolar correspondente à idade, diferenciando-se do grupo de crianças adiantadas na escola do estudo de Graminha (1992).

Outro dado de destaque diz respeito à incidência de dificuldades de fala. Tanto as avaliações das mães quanto das professoras apontaram para a maior ocorrência dessas dificuldades entre as crianças do grupo BRA, o que se assemelha aos resultados de Graminha (1992), Marturano, Magna e Murtha (1992) e Magna (1997), que também encontraram resultados significativos quanto à presença de dificuldades de fala/linguagem associadas a problemas de aprendizagem.

Os resultados quanto às dificuldades de fala presentes no grupo com baixo rendimento sugerem que estas dificuldades constituem-se em fator de risco significativo para o desenvolvimento de problemas na aprendizagem acadêmica, indo na mesma direção dos resultados do estudo de Needleman et al. (1996), realizado para detectar fatores de risco relacionados com a presença de transtornos de comportamento e aprendizagem em alunos do ensino fundamental. Os autores encontraram uma associação positiva e significativa dos dois tipos de transtornos com dificuldades na expressão e compreensão da linguagem e concluíram que dificuldade na compreensão da linguagem é preditora de transtornos de aprendizagem.

Tais dados vão ao encontro da hipótese anteriormente levantada de que as crianças do grupo BRA se encontram em desvantagem de desenvolvimento frente às crianças do grupo ARA, além da desvantagem acadêmica.

Ainda com relação ao comportamento das crianças, chamam a atenção os resultados da Escala B de Rutter (respondida pelos professores), uma vez que na maioria dos itens houve diferença significativa entre os grupos BRA e ARA, quase sempre (exceção de um único item: preocupado/preocupa-se com muitas coisas) com maior incidência para o grupo BRA.

Isto sugere que, principalmente no que diz respeito a comportamento, os professores provavelmente discriminam melhor entre os dois grupos e, também, aponta para a forte associação entre dificuldades de aprendizagem e problemas de comportamento. Barra, Toledo e Rodriguez (2002), analisando a opinião dos professores sobre o rendimento acadê- mico, o comportamento de escolares e sobre as necessidades das crianças de serviços especializados por não estarem respondendo adequadamente às demandas da escola, concluíram que os professores avaliam melhor problemas de comportamento do que o baixo rendimento acadêmico dos alunos, tendo uma alta sensibilidade para detectar crianças com problemas emocionais/comportamentais.

De forma semelhante, Del Prette e Del Prette (1998a), em um estudo que comparava crianças com e sem dificuldades de aprendizagem com relação às habilidades sociais, a partir da avaliação de diferentes informantes (professores, colegas e a própria criança), observaram que o professor é quem mais diferenciava os dois grupos de crianças quanto às características interpessoais.

Nos resultados obtidos por Machado, Marturano, Loureiro, Linhares e Bessa (1994), embora focalizassem em especial o comportamento em sala de aula, também fica evidente, na avaliação feita pelos professores, que o grupo com dificuldades de aprendizagem apresentava mais problemas de comportamento, principalmente aqueles comportamentos ligados a problemas de externalização, reforçando ainda mais a idéia de que as crianças que apresentam dificuldades de aprendizagem também são avaliadas como tendo mais problemas comportamentais.

Embora não seja possível, pelos resultados deste estudo, identificar a ordem de ocorrência das dificuldades, podese supor que a associação entre problemas de comportamento e de aprendizagem seja uma via de mão dupla em que um problema se apresenta como condição de risco para o outro.

Os resultados também sugerem que as crianças com baixo rendimento acadêmico podem ter problemas no relacionamento com os colegas, uma vez que, com maior freqüência, não são queridas pelos colegas e brigam muito com outras crianças. Isso, provavelmente, restringe a possibilidade de se integrarem com crianças que apresentam comportamentos mais positivos ou menos disruptivos, dificultando de certa forma o aprendizado de comportamentos mais adequados ou, ainda, favorecendo o isolamento destas crianças, o que pode prejudicar ainda mais a aprendizagem e seu desenvolvimento em geral. A literatura aponta que crianças com dificuldades de aprendizagem costumam ser descritas como tendo mais dificuldades de relacionamento e tendendo a serem mais inquietas, briguentas, inibidas e sem iniciativa (Maluf \& Bardelli, 1991).

Numa revisão de literatura, Del Prette e Del Prette (1998b) relatam vários estudos os quais mostram que as dificuldades nos relacionamentos interpessoais de crianças com dificuldades de aprendizagem incluem uma tendência a apresentarem interações mais negativas com os colegas e a serem mais agressivas, mais imaturas, com mais problemas de personalidade, com menos comportamentos orientados para a tarefa e com repertório mais restrito de comportamentos interpessoais adequados.

Na prática, os resultados deste estudo salientam que ao trabalhar com crianças com problemas na aprendizagem ou baixo rendimento acadêmico é necessário dar atenção tanto 
às questões diretamente ligadas à aprendizagem quanto às dificuldades emocionais e comportamentais apresentadas pela criança, destacando-se ainda a premência de um trabalho que facilite o desenvolvimento de habilidades sociais e uma melhor integração com os pares.

\section{Referências}

Arnold, E. M, Goldston, D. B., Walsh, A. K., Reboussin, B. A., Daniel, S. S., Hickman, E., \& Wood, F. B. (2005). Severity of emotional and behavioral problems among poor and typical readers. Journal of Abnormal Child Psychology, 33(2), 205-217.

Barbaresi, W. J., Katusic, S. K., Colligan, R. C., Weaver, A. L., \& Jacobsen, S. J. (2005). Math learning disorder: incidence in a population-based birth cohort, 1976-82. Ambulatory Pediatrics, 5(5), 281-289.

Barra, F. de la, Toledo, V., \& Rodriguez, J. (2002). Estúdio de salud mental em dos cohorte de niños escolares de Santiago occidente II: factores de riesgo familiares y escolares. Revista Chilena de Neuro-Psiquiatria, 40, 347-360.

Clawson, A. (1980). Bender infantil: manual de diagnóstico clínico. Porto Alegre: Artes Médicas.

Del Prette, A., \& Del Prette, Z. A. P. (1998a). Habilidades sociales y dificultades de aprendizaje: algunas cuestiones [Resumo]. In World Congress Committee (Org.), $2^{\text {nd }}$ World Congress of Behavior and Cognitive Therapies. Resumos (p. 86). Acapulco: Autor.

Del Prette, A., \& Del Prette, Z. A . P. (1998b). Desenvolvimento interpessoal e educação escolar: o enfoque das habilidades sociais. Temas em Psicologia, 6(3), 205-215.

Farran, D. C., \& Cooper, D. H. (1986). In D. C. Farran \& J. D. McKinney (Orgs.), Risk in intellectual and psychosocial development (pp. 187-226). Orlando: Academic.

Finnstrom, O., Gaddlin, P. O., Leijon, I., Samuelsson, S., \& Wadsby, M. (2003). Very-low-birth-weight children at school age: academic achievement, behavior and self-esteem and relation to risk factors. Journal of MaternalFetal \& Neonatal Medicine, 14(2), 75-84.

Gordon, N. (1993). Learning disorders and delinquency. Brain \& Development, 15(3), 169-172.

Graminha, S. S. V. (1992). Problemas emocionais/comportamentais e nível de escolaridade da criança. Arquivos Brasileiros de Psicologia, 44(1/2), 74-92.

Graminha, S. S. V. (1994) A Escala Comportamental Infantil de Rutter A2: estudos de adaptação e fidedignidade. Estudos de Psicologia (Campinas), 11(3), 34-42.

Graminha, S. S. V. (1998). Recursos metodológicos para pesquisas sobre riscos e problemas emocionais e comportamentais na infância. In G. Romanelli \& Z. M. M. Biasoli-Alves (Orgs.), Diálogos metodológicos sobre práticas de pesquisa (pp. 71-86). Ribeirão Preto: Legis Summa.

Graminha, S. S. V., Martins, M. A. O., \& Miura, R. T. (1996). Fatores potenciais de risco em crianças com problemas emocionais/comportamentais. Psico, 7(2), 53-79.

Haager, D., \& Vaughn, S. (1995). Parent, teacher, peer, and self-reports of the social competence of students with learning disabilities. Journal of Learning Disabilities, 28(4), 205-215, 231.

Hinshaw, S. P. (1992). Externalizing behavior problems and academic underachievement in childhood and adolescence: causal relationships and underlying mechanisms. Psychological Bulletin, 111, 127-155.

Kolvin, I., Miller, F. J. W., Fleeting, M., \& Kolvin, P. A. (1988). Risk/ protective factors for offending with particular reference to deprivation. In M. Rutter (Org.), Studies of psychosocial risk: the power of longitudinal data (pp. 77-95). Cambridge: Cambridge University Press.

Lewis, R. J., Dlugokinski, E. L., Caputo, L. M., \& Griffin, R. B. (1988). Children at risk for emotional disorders: risk and resource dimensions. Clinical Psychology Review, 8, 417-440.
Machado, V. L. S., Marturano, E. M., Loureiro, S. R., Linhares, M. B. M., \& Bessa, L. C. D. (1994). Crianças com dificuldades na aprendizagem escolar: características de comportamento conforme avaliação de pais e professores. Arquivos Brasileiros de Psicologia, 46(3/4), 184-198.

Magna, J. M. (1997). Atraso escolar: um levantamento de condições de desenvolvimento antecedentes à entrada na primeira série. Dissertação de mestrado não-publicada, Faculdade de Medicina de Ribeirão Preto da Universidade de São Paulo, Ribeirão Preto.

Maluf, M. R., \& Bardelli, C. (1991). As causas do fracasso escolar na perspectiva de professoras e alunos de uma escola de primeiro grau. Psicologia: Teoria e Pesquisa, 7, 255-262.

Marturano, E. M., Linhares, M. B. M., \& Parreira, V. L. C. (1993). Problemas emocionais e comportamentais associados a dificuldades na aprendizagem escolar. Medicina Ribeirão Preto, 26(2), 161-175.

Marturano, E. M., Magna, J. C., \& Murtha, P. C. (1992). Contribuição ao diagnóstico das dificuldades de aprendizagem escolar. Revista Psicopedagogia, 11(24), 7-15.

Marturano, E. M., Magna, J. C., \& Murtha, P. C. (1993). Procura de atendimento psicológico para crianças com dificuldades escolares: um perfil da clientela. Psicologia: Teoria e Pesquisa, 9(1), 207-226.

Maughan, B. (1988). School experiences as risk/protective factors. In M. Rutter (Org.), Studies of psychosocial risk: the power of longitudinal data (pp. 200-220). Cambridge: Cambridge University Press.

Medeiros, P. C., \& Loureiro, S. R. (2004). A observação clínica do comportamento de crianças com queixa de dificuldade de aprendizagem. In E. M. Marturano, M. B. M. Linhares, \& S. R. Loureiro (Orgs.), Vulnerabilidade e proteção: indicadores na trajetória de desenvolvimento do escolar (pp. 107-136). São Paulo: Casa do Psicólogo; FAPESP.

Meister, E. K., Bruck, I., Antoniuk, S. A., Crippa, A. C., Muzzolon, S. R., Spessato, A., \& Gregolin, R. (2001). Learning disabilities: analysis of 69 children. Arquivos de Neuropsiquiatria, 59(2-B), 338-341.

Needleman, C. A., Salamanco, M. G., Gil, C., Murno, J. R., Lamy, P., \& De Gentile, A. S. (1996). Abordaje interdisciplinario de la problemática del escolar. Archives Argentinos de Pediatria, 94(3), 159-173.

Okano, C. B., Loureiro, S. R., Linhares, M. B. M., \& Marturano, E. M. (2004). Crianças com dificuldades escolares atendidas em programa de suporte psicopedagógico na escola: avaliação do autoconceito. Psicologia: Reflexão e Crítica, 17(1), 121-128.

Raven, J. C., Raven, J., \& Court, J. H. (1988). Matrizes Progressivas Coloridas: Escala Especial. São Paulo: Casa do Psicólogo.

Roeser, R. W., \& Eccles, J. S. (2000). Schooling and mental health. In A. J. Sameroff, M. Lewis, \& S. M. Miller (Orgs.), Handbook of developmental psychopathology (pp. 135-156). Nova York: Kluwer; Plenum.

Rutter, M. (1967). A children's behavior questionnaire for completion by teachers: preliminary findings. Journal of Child Psychology and Psychiatry, 8, 1-11.

Rutter, M. (1987). Psychosocial resilience and protective mechanisms. American Journal of Orthopsychiatry, 57(3), 316-331.

Rutter, M., Caspi, A., Fergusson, D., Horwood, l. J., Goodman, R., Maughan, B., Moffitt, T. E., Meltzer, H., \& Carroll, J. (2004). Sex differences in developmental reading disability: new findings from 4 epidemiological studies. Journal of American Medical Association, 291(16), 2007-2012.

Santos, P. L. (2002). Riscos e recursos em crianças com baixo e alto rendimento acadêmico - um estudo comparativo. Tese de doutorado nãopublicada, Faculdade de Filosofia, Ciências e Letras de Ribeirão Preto, Universidade de São Paulo, Ribeirão Preto.

Santos, P. L., \& Graminha, S. S. V. (2005). Estudo comparativo das características do ambiente familiar de crianças com alto e baixo rendimento acadêmico. Paidéia, 15(31), 217-226.

Stevanato, I. S., Loureiro, S. R., Linhares, M. B. M., \& Marturano, E. M. (2003). Autoconceito de crianças com dificuldades de aprendizagem e problemas de comportamento. Psicologia em Estudo, 8(1), 67-76. 
Silver, L. B. (1981). The relationship between learning disabilities, hyperactivity, distractibility and behavioral problems. Journal of American Academy of Child Psychiatry, 20, 385-397.

Speakman, N. J., Herman, K. L., \& Vogel, S. A. (1993). Risk and resilience in individuals with learning disabilities: a challenge to the field. Learning Disabilities: Research \& Practice, 8(1), 59-65.

Stein, L. M. (1994). TDE - Teste de Desempenho Escolar. São Paulo: Casa do Psicólogo.
Thompson, J. R. J., Lampron, L. B., Johanson, D. F., \& Eckstein, T. L. (1990). Behavior problems in children with the presenting problem of poor school performance. Journal of Pediatric Psychology, 15, 3-29.

Vickers, H. S. (1994). Young children at risk: differences in family functioning. Journal of Educational Research, 87(5), 262-270.

Witt, W. P., Riley, A. W., \& Coiro, M. J. (2003). Childhood functional status, family stressors, and psychosocial adjustment among schoolaged children with disabilities in the United States. Archives of Pediatrics and Adolescent Medicine, 157(7), 687-695.

Patrícia Leila dos Santos, doutora em Psicologia pela Universidade de São Paulo - Ribeirão Preto, é professora no Departamento de Neurologia. Psiquiatria e Psicologia Médica, Faculdade de Medicina, Universidade de São Paulo - Ribeirão Preto. Endereço para correspondência: Rua Tenente Catão Roxo, 2650 (Monte Alegre); Ribeirão Preto, SP; CEP 14051-140. Tel.: (16) 3602- 4608. Fax: (16) 3602-4504. E-mail: plsantos@fmrp.usp.br

Sônia Santa Vitaliano Graminha, doutora em Psicologia, pela Universidade Estadual Paulista - Franca, SP, é professora no Departamento de Psicologia e Educação, Faculdade de Filosofia, Ciências e Letras de Ribeirão Preto, Universidade de São Paulo. E-mail: ssvgrami@usp.br 